\title{
Presencia de Haemagogus equinus Theobald, 1903 (Diptera: Culicidae) en los municipios de Soledad y Malambo en el departamento del Atlántico, Colombia, 1998-2005
}

\author{
Ronald Maestre-Serrano1, Consuelo Vergara-Sanchez¹, Guillermo Berrueco-Rodríguez¹, \\ Betsy Bello-Novoa², Helena Brochero ${ }^{2,3}$ \\ 1 Laboratorio Departamental de Salud Pública, Secretaría de Salud del Atlántico, Barranquilla, Colombia \\ 2 Laboratorio de Entomología, Red Nacional de Laboratorios, Instituto Nacional de Salud, Bogotá, D.C., \\ Colombia \\ 3 Facultad de Agronomía, Universidad Nacional de Colombia, Bogotá, D.C., Colombia
}

Introducción. La fiebre amarilla es una enfermedad de interés en salud pública. Es transmitida por mosquitos de los géneros Haemagogus spp. y Sabethes spp., en su ciclo selvático, y Aedes aegypti en su ciclo urbano. Para Colombia se consideran vectores eficientes de esta patología las especies Haemagogus janthinomys y Haemagogus equinus.

Objetivo. Registrar la presencia del mosquito $H$. equinus, considerado vector eficiente de fiebre amarilla selvática en Colombia, en áreas periurbanas de los municipios de Soledad y Malambo en el departamento del Atlántico, desde 1998 a 2005.

Materiales y métodos. Se empleó la información de los registros del material entomológico recolectado de 14 larvitrampas ubicadas en el Aeropuerto Ernesto Cortissoz del municipio de Soledad entre 1997 y 2005 y 10 larvitrampas ubicadas en el Batallón Vergara y Velasco del municipio de Malambo durante el 2005.

Resultados. En Soledad, H. equinus se registró por primera vez en 1998. Hasta el 2005, se registraron 197 larvas. Los ejemplares se encontraron compartiendo hábitat con A. aegypti Linnaeus, 1762, Culex nigripalpus Theobald, 1901 y Uranotaenia lowii Theobald, 1901. En Malambo, el primer hallazgo ocurrió en el 2005, registrándose durante todo el año 641 larvas no asociadas a otros culícidos.

Conclusión. La presencia de $H$. equinus en larvitrampas ubicadas en áreas cercanas al casco urbano, evidencia adaptaciones a criaderos artificiales, riesgo de urbanización, que sumado a los altos índices de infestación de $A$. aegypti y la potencial introducción del virus de la fiebre amarilla a través de este importante puerto, constituyen riesgo para esta patología en la zona.

Palabras clave: fiebre amarilla/epidemiología, Aedes aegypti, vectores de enfermedades, vigilancia epidemiológica, Colombia.

Presence of Haemagogus equinus Theobald, 1903 (Diptera: Culicidae), in Soledad and Malambo, in the Province of Atlántico, Colombia

Introduction. Yellow fever is a serious illness public health importance and is transmitted by mosquitoes of the genera Haemagogus and Sabethes in the rural and forest environments, and by Aedes aegypti in the urban setting. In Colombia, Haemagogus janthinomys and $\mathrm{H}$. equinus are considered efficient vectors of this viral disease.

Objective. The presence of the mosquito Haemagogus equinus was recorded over an 8 year period, in the periurban areas of the Soledad and Malambo municipalities (Atlantico Province) of northern Colombia.

Materials and methods. The data was obtained from records of the entomological collections from two collection sites: (1) 14 larva traps located at the Erneasto Cortissoz airport in the municipality of Soledad between 1997-2005 and (2) 10 larva traps located at Vergara and Velasco Batallion in the municipality of Malambo in 2005.

Results. Haemogogus equinus was reported for the first time in Soledad in 1998. In the following 8 years, 197 larvae were reported. The individuals were found sharing the trap with 


\begin{abstract}
Aedes aegypti, Culex nigripalpus and Uranotaenia lowii. In Malambo, the first discovery of H.equinus occurred in 2005, with a total of 641 larvae. No other Culicidae were associated with it. Conclusion. The presence of $H$. equinus in larvitraps located near the urban zone, shows adaptation to the use of artificial containers as larval habitats, Urbanization of this species in zones with a high $A$ e. aegypti infestation index increases the potential introduction of sylvan yellow fever virus and constitutes a risk for re-emergence of urban cycles of yellow fever.
\end{abstract}

Key words: Yellow fever/epidemiology, Aedes aegypti, disease vectors, epidemiologic surveillance, Colombia.

La fiebre amarilla es una enfermedad altamente letal transmitida por vectores. Se comporta de forma endémica y causa brotes aislados en humanos en zonas tropicales de África y América (1-3). El virus de la fiebre amarilla pertenece al género Flavivirus de la familia Flaviviridae. Es un virus ARN monocatenario, que se mantiene en dos ciclos básicos (urbano y selvático) y es transmitido por la picadura de mosquitos hematófagos de la familia Culicidae. Se han incriminado como vectores en Colombia especies de los géneros Haemagogus spp. y Sabethes spp., en su ciclo selvático, y Aedes aegypti en su ciclo urbano (4-6). Los mosquitos permanecen infectados durante toda su vida. También se ha determinado que pueden transmitir el virus a su descendencia, lo cual podría explicar el mecanismo que permite, durante largos periodos de sequía, mantener el virus cuando no hay la cantidad suficiente de vectores para asegurar la transmisión (7-9).

En América se han descrito 28 especies del género Haemagogus, de las cuales, nueve se han registrado para Colombia y dos se consideran de interés en salud pública, por considerarse vectores eficientes del virus de la fiebre amarilla selvática: Haemagogus janthinomys Dyar, 1921 y Haemagogus equinus Theobald, 1903 (Diptera: Culicidae) (10-15).

$H$. equinus es un mosquito cuya distribución geográfica en el mundo se restringe al área neotropical. Esta especie se ha reportado en todos

\footnotetext{
Correspondencia:

Ronald Maestre Serrano, Laboratorio Departamental de Salud Pública, Secretaria de Salud del Atlántico, Calle $75 \mathrm{~N}^{\circ}$ 72-140, Barranquilla, Colombia. rmaestre22@yahoo.com
}

Recibido: 29/05/07; aceptado: 17/10/07 los países centroamericanos, en algunas regiones antillanas, como Jamaica, en Estados Unidos y en Suramérica con registros en Colombia, Venezuela y Brasil (11-17).

En Colombia, $H$. equinus se ha registrado entre los 2 y 1.007 metros sobre el nivel del mar (msnm), en departamentos como Antioquia, Cesar, Córdoba, Cundinamarca, La Guajira, Huila, Magdalena, Santander, Tolima y Meta $(16,17)$. Se considera una especie silvestre, restringida a las áreas selváticas y rurales con predominio de bosques primarios, secundarios y de galería $(10,18)$, donde sus formas inmaduras se desarrollan en una variedad de hábitats como huecos de los árboles, axilas de hojas, troncos cortados de bambú y huecos de rocas $(11,18)$. Los adultos viven en la copa de los árboles y en horas diurnas ejercen sus actividades de alimentación, reposo y reproducción. Las hembras se alimentan de la sangre de los animales que viven en los árboles, como los primates no humanos del género Alouatta, y así mantienen el ciclo selvático de transmisión del virus de la fiebre amarilla $(10,17,19)$.

Sin embargo, en el área metropolitana de Bucaramanga (Santander), se registró por primera vez su presencia en criaderos artificiales (llantas) $(11,16,20) \mathrm{y}$, recientemente, se encontraron mosquitos de este género con hábitos de picadura dentro y fuera de las viviendas en un área rural (Soto H, López E, Florián J, Olano V. Evaluación entomológica sobre vectores de fiebre amarilla selvática en el municipio de Valledupar, Cesar. Memorias, XII Congreso Colombiano de Parasitología y Medicina Tropical. Biomédica. 2005;25(Supl.1):165-6). Estos hallazgos han sugerido exitosos procesos de adaptación a la presión antrópica incrementando el contacto hombre-vector. 
En el departamento del Atlántico se encuentran ubicados importantes puertos marítimos, fluviales y aéreos de Colombia. Está ubicado en el mar Caribe, recibe la desembocadura del río Magdalena, concentra grandes poblaciones humanas en los municipios de Soledad y Malambo, y a través del aeropuerto internacional recibe viajeros de departamentos y otros países donde la fiebre amarilla es endémica. Por lo anterior, se considera zona de riesgo para las patologías de transmisión vectorial, por ser potencial para la introducción de especies no nativas y con interés en salud pública como es el caso de la especie Aedes albopictus. Debido a esto, desde 1998 se implementó la vigilancia entomológica centinela por larvitrampa, en los puertos aéreos, marítimos y terrestres, como uno de los componentes fundamentales dentro del programa de prevención de enfermedades transmitidas por vectores.

El presente artículo tiene como objetivo registrar la presencia de $H$. equinus en áreas periurbanas de los municipios de Soledad y Malambo a través de larvitrampas durante los años 1998 a 2005.

\section{Materiales y métodos}

\section{Área de estudio}

El municipio de Soledad se encuentra ubicado en el área metropolitana de Barranquilla a $10^{\circ} 55^{\prime}$ latitud norte y $74^{\circ} 46^{\prime}$ longitud oeste; tiene una altitud de $5 \mathrm{msnm}$ y una extensión de $66 \mathrm{~km}^{2}(21)$. Con temperatura media anual de $28^{\circ} \mathrm{C}$, un promedio anual de precipitación de $1.127 \mathrm{~mm}$ y entre $75 \%$ y $85 \%$ de humedad relativa, registra un clima semiárido $(21,22)$. Soledad no posee bosques naturales, pero sí hectáreas de suelo agrícola y pastizal.

El municipio de Malambo se encuentra ubicado a $10^{\circ} 52^{\prime}$ de latitud norte y $74^{\circ} 47^{\prime}$ de longitud oeste, al nororiente del departamento del Atlántico, distante a $12 \mathrm{~km}$ de la ciudad capital (Barranquilla) y a un kilómetro del Aeropuerto Ernesto Cortissoz (23) (figura 1). Con una extensión territorial de 99 $\mathrm{km}^{2}$, limita al norte con el municipio de Soledad. Su clima es húmedo tropical con una altitud de $10 \mathrm{msnm}$, con una temperatura promedio anual de $28{ }^{\circ} \mathrm{C}$ y una humedad relativa anual entre $80 \%$ y $84 \%(22,23)$. El municipio de Malambo, tiene 90 hectáreas de bosque natural, 500 hectáreas dedicadas a prácticas agrícolas, 6.909 hectáreas de pastos y 285 hectáreas correspondientes al área urbana (24).

\section{Ubicación de larvitrampas y recolección de material entomológico}

La larvitrampa consistió en un recipiente fabricado a partir de llanta de automóvil. La llanta se cortó, dejando una superficie cóncava que constituye el interior de la larvitrampa. Cada larvitrampa fue etiquetada con un número único consecutivo y el logotipo de la Secretaría de Salud del Atlántico. Todas las larvitrampas se colocaron a un metro de altura sobre el nivel del suelo, en lugares protegidos de la luz directa del sol, de las aguas lluvias y de la perturbación por animales y personas. En su interior se colocó agua declorada y un trozo de madera con una superficie rugosa que facilitó la postura de huevos de los mosquitos (25).

En 1997, se implementó el primer punto de vigilancia entomológica, correspondiente al Aeropuerto Ernesto Cortissoz del municipio de Soledad, ubicando 14 larvitrampas en tres zonas: cinco en los jardines externos, dos en el acueducto y siete en la base de mantenimiento de Avianca. Su función principal era vigilar la introducción de A. albopictus (Skuse, 1894).

En enero de 2005, se ubicaron 10 larvitrampas en el Batallón Vergara y Velasco del municipio de Malambo con la siguiente distribución: dos cercanas a vegetación de matorral espinoso, cinco cerca a viviendas y oficinas, y tres cerca a la ciénaga; se informó con anterioridad a las autoridades acerca de la ubicación, el objetivo, el uso, la frecuencia y el responsable de la inspección. El objetivo de su instalación en este punto estuvo justificado por la gran movilización de tropas de este batallón desde diferentes áreas endémicas para enfermedades transmitidas por vectores en el país, y por la detección de una posible extensión en la población de $H$. equinus, ya detectada en el departamento; la adaptación de mosquitos del género Anopheles, responsables del paludismo, a criaderos hechos por el hombre y la ubicación de otro punto de vigilancia para la introducción de $A$. albopictus. 
La vigilancia entomológica mediante larvitrampas se implementó de forma complementaria a la inspección entomológica en otros criaderos existentes en cada área. Las muestras entomológicas recolectadas se empacaron en frascos de $50 \mathrm{ml}$ con agua de la misma larvitrampa, indicando lugar y fecha de recolección, número de larvitrampa, nombre del recolector y cantidad de ejemplares. Éstas se transportaron a la Unidad de Entomología del Laboratorio Departamental de Salud Pública del Atlántico.

Las larvas se fijaron en alcohol al $70 \%$ y las pupas se mantuvieron en el insectario en condiciones controladas de temperatura, humedad relativa, fotoperiodo $12: 12$ y condiciones de bioseguridad, hasta emerger los adultos. Todos los especímenes se procesaron en montaje permanente y se determinaron taxonómicamente a partir de caracteres morfológicos, usando micrómetro ocular en microscopio y estereomicroscopio, y siguiendo las claves taxonómicas disponibles (10,25-27). Un porcentaje de los ejemplares recolectados se incluyó en la colección de referencia del departamento del Atlántico. El Laboratorio de Entomología del Instituto Nacional de Salud realizó la confirmación de la determinación taxonómica en todos los casos.

\section{Análisis estadístico}

Se aplicó una prueba t para analizar si había diferencias significativas en los promedios mensuales del número de larvas registradas de $H$. equinus, en las áreas estudiadas durante el año 2005. Se estableció un nivel de significancia de $p=0,05$. Los datos se procesaron con ayuda del paquete estadístico SPSS.

\section{Resultados}

En el municipio de Soledad se detectó por primera vez la presencia de $H$. equinus en julio de 1998. Desde ese año hasta el 2005, se registraron 197 larvas de segundo y tercer estadio en las 14 larvitampas ubicadas en este punto. La distribución del número de larvas en relación con la ubicación de larvitrampas en Soledad, correspondió a: 64 en jardines externos, 48 en acueducto y 85 para base de mantenimiento de Avianca (figura 1).
Durante este período, los meses de julio a noviembre se caracterizaron por registrar la presencia permanente de $\mathrm{H}$. equinus; en octubre de 2002 se recolectó el mayor número de especímenes (25 larvas), seguido de noviembre de 2003 (24 larvas) y julio de 2005 (20 larvas) (figura 2). Las larvas de $H$. equinus se encontraron compartiendo el hábitat con otras especies de culícidos como Culex nigripalpus, Uranotaenia lowiiy $A$. aegypti. En este municipio se suspendió la vigilancia entomológica mediante larvitrampas durante el 2001, razón por la cual no se obtuvo reconocimiento de culícidos para este año.

En el municipio de Malambo se registró $H$. equinus por primera vez en junio de 2005. Durante el segundo semestre de este año, se recolectaron 641 larvas de esta especie, distribuidas de la siguiente forma: 42 larvas para la larvitrampa $\mathrm{N}^{\circ}$ 5 y 599 larvas para la larvitrampa $N^{\circ} 6$ (figura 1). Éstas nunca se encontraron compartiendo hábitat con otros culícidos. Las ocho larvitrampas restantes no reportaron positividad para $H$. equinus pero sí para otras especies de Culícidos, tales como A. albimanus Wiedemann 1820, $A$. aegypti, Culex coronator Dyar y Knab, 1906 y Culex nigripalpus. Durante el periodo estudiado, de julio a noviembre se caracterizaron por registrar el mayor número de larvas de $H$. equinus: julio (187 larvas), agosto (197 larvas) y octubre (146 larvas) (figura 2). La prueba t evidenció diferencias significativas ( $p=0,03 ; g l=7 ; n=8)$ en el promedio mensual de larvas de $H$. equinus registradas para el municipio de Soledad y Malambo durante el año 2005.

\section{Discusión}

Hasta 1998 el departamento del Atlántico no había registrado la presencia de $H$. equinus. Este hallazgo constituyó un resultado indirecto del sistema de vigilancia entomológica centinela para la especie $A$. albopictus implementado en el Aeropuerto Ernesto Cortissoz del municipio de Soledad desde 1997. H. equinus registró una adaptación positiva a este ambiente y una expansión de la población a nuevas áreas geográficas, como se evidenció con el reconocimiento de esta especie en larvitrampas del municipio de Malambo, triplicando en seis 


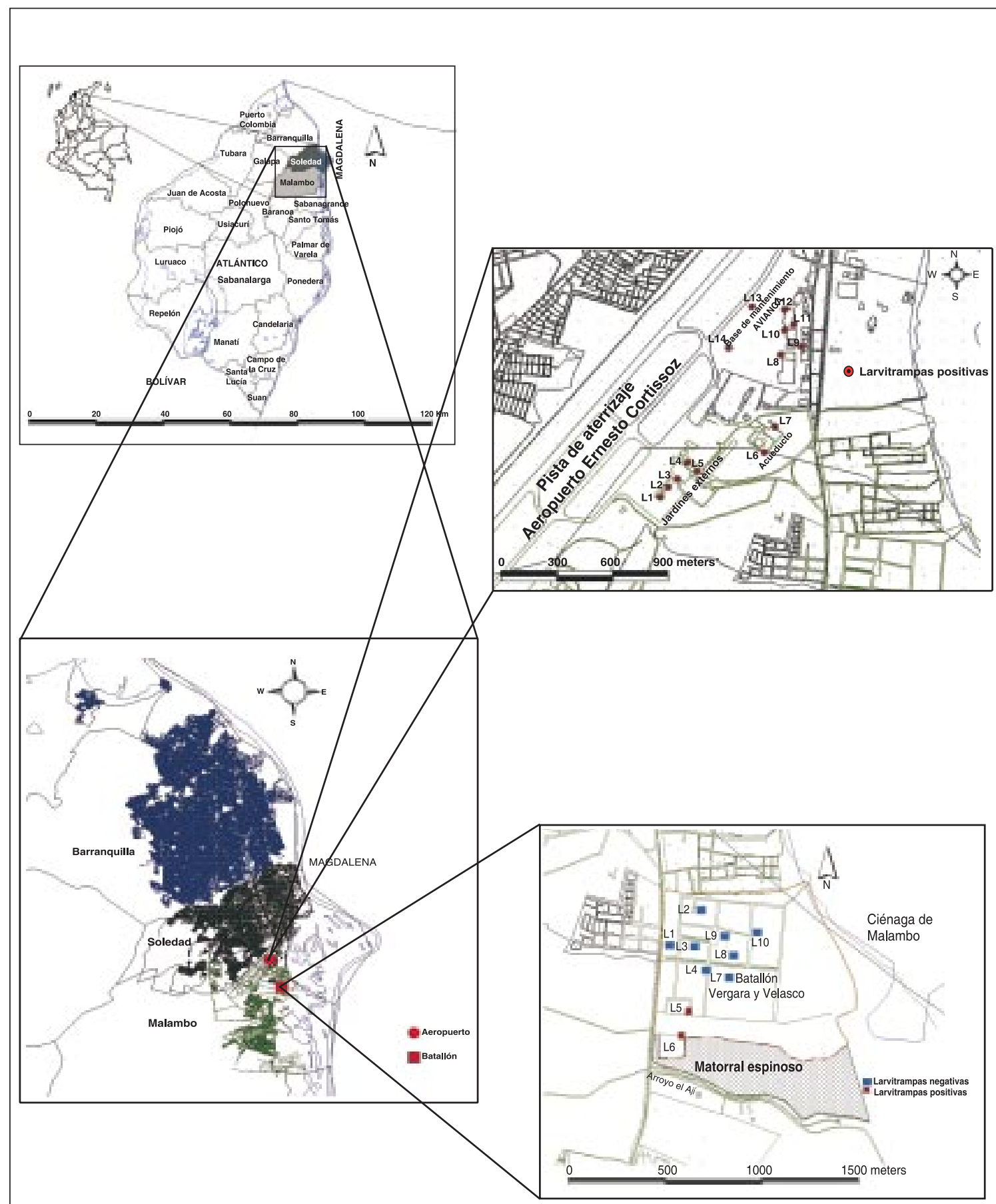

Figura 1. Ubicación de las larvitrampas positivas para Haemagogus equinus en el aeropuerto Ernesto Cortissoz (municipio de Soledad) y Batallón Vergara y Velasco (municipio de Malambo) en el departamento del Atlántico. 


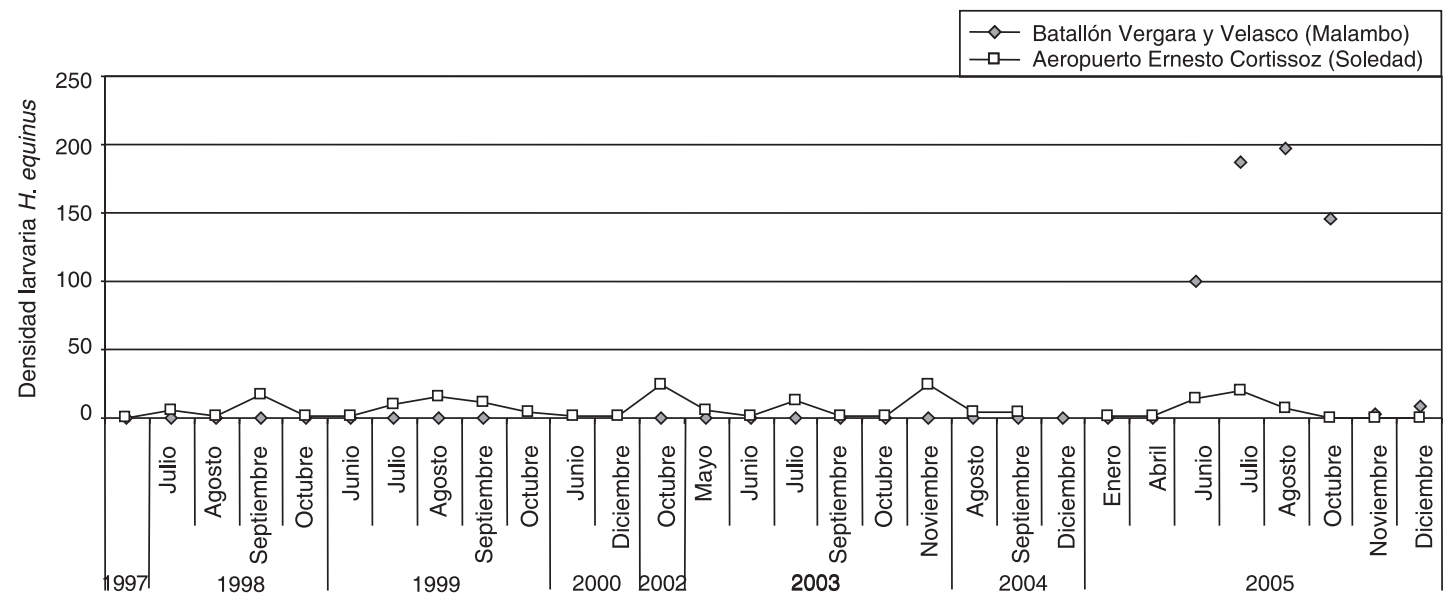

Figura 2. Registro del número de larvas de $H$. equinus en el aeropuerto Ernesto Cortissoz - Soledad (1998-2005) y batallón Vergara y Velasco - Malambo (2005) en el departamento del Atlántico.

meses el número de individuos recolectados con respecto al reportado en ocho años para el municipio de Soledad; esto se observa en los registros para el 2005 , en los cuales se encontraron diferencias significativas en los promedios de los individuos recolectados entre las dos localidades $(p=0,03 ; g l=7 ; n=8)$. En Malambo, las larvitrampas que registraron la especie se encontraban a menos de cinco metros de distancia de un área con abundancia de vegetación cactácea espinosa (hábitat apto para su éxito reproductivo) $(23,24)$. De hecho, las larvitrampas de Malambo en las que no se encontró $H$. equinus estuvieron ubicadas en jardines y zonas de mayor presión antrópica (figura 1).

Morales (1968) y Morales et al. (1984), encontraron larvas de $H$. equinus junto a larvas de $A$. aegypti en huecos de árboles. Olano et al. (1997) reportaron por primera vez para Colombia, la presencia de $H$. equinus en criaderos artificiales (llantas) en el aeropuerto Palonegro, el Jardín Botánico, el Hospital San Juan de Girón y el Cementerio Las Colinas, en el área metropolitana de Bucaramanga (Santander). Los resultados de este estudio coinciden con estos hallazgos y confirman que $H$. equinus se ha ido adaptando a criaderos artificiales, ampliando su rango de distribución. Se evidencia, además, que $H$. equinus logra compartir el hábitat con otros culícidos y puede, incluso, desplazarlos y volverse la especie dominante, como se encontró en Malambo.

Recientemente, se registraron mosquitos adultos de $H$. janthinomys Dyar, 1.921 con actividad de picadura intradomicilar y formas inmaduras de Haemagogus lucifer Howard, Dyar \& Knab, y 1.912 en criaderos artificiales en el peridomicilio de viviendas en las veredas Punta del Este (corregimiento Aguas Blancas) y Gallinetas (corregimiento María Angola) del municipio de Valledupar en el departamento del Cesar (Soto H, López E, Florián J, Olano V. Evaluación entomológica sobre vectores de fiebre amarilla selvática en el municipio de Valledupar, Cesar. Memorias, XII Congreso Colombiano de Parasitología y Medicina Tropical. Biomédica. 2005;25 (Supl.1):165-6).

Estos procesos se han identificado para el país en otros culícidos, tales como anofelinos (28) (Brochero H, Mejía R, Chacón R, Córdoba S, Palacio J, Arévalo $\mathrm{C}$, et al. Artificial containers: ¿New breeding places for malaria vectors? Memorias, XVI International Congress for Tropical Medicine and Malaria. 2000;2:28). Evidentemente, estos factores, aunados a la gran movilidad de personas infectadas y susceptibles, constituyen un importante riesgo de surgimiento 
de enfermedades transmitidas por vectores. Además, una vez adaptadas estas especies a criaderos artificiales, existe la posibilidad de urbanización. Para el departamento del Atlántico, $H$. equinus se encontró en larvitrampas ubicadas a menos de $500 \mathrm{~m}$ del casco urbano donde se concentra la mayor parte de la población. Estos municipios registran altos índices de infestación para $A$. aegypti, desplazamiento de población militar del batallón Vergara y Velasco desde zonas endémicas para el virus de la fiebre amarilla, como la Sierra Nevada de Santa Marta, y asentamientos de población desplazada por aspectos relacionados con el conflicto armado, lo que determina una mayor alarma para la aparición de la fiebre amarilla en el departamento.

En este estudio se observó un incremento en el número de larvas de $H$. equinus encontradas en larvitrampas durante los meses de julio a noviembre, lo cual es coincidente con la distribución de la precipitación de tipo monomodal, cuyo período va de mayo a octubre, caracterizándose septiembre como el mes más lluvioso $(22,24)$. Los registros de esta especie en diciembre pueden atribuirse a la influencia del periodo de transición de lluvia a seco, en los meses de diciembre a enero.

En Colombia, el virus de la fiebre amarilla ha mantenido circulación en diferentes regiones del país. Desde el 2000 hasta la semana epidemiológica 52 de 2005, se confirmaron 188 casos de fiebre amarilla en Colombia (29-31). Entre ellos se pueden citar el brote de la región del Catatumbo (Norte de Santander) en la semana 28 de 2003 y el ocurrido en la Costa Atlántica durante la semana epidemiológica 1 del 2004.

Teniendo presente los hallazgos reportados por Olano et al. (1997) para el departamento de Santander y los encontrados en el presente trabajo, se pone de manifiesto la necesidad de implementar el sistema de vigilancia entomológica activa basado en larvitrampas, particularmente en áreas endémicas para fiebre amarilla, puertos marítimos, aéreos y zonas de concentración militar, como batallones (32). Este estudio evidencia que las larvitrampas constituyen un apoyo a la vigilancia en salud pública de enfermedades transmitidas por vectores, que permite detectar la introducción y adaptación de especies de interés médico a un área geográfica de interés y, por tanto, definir estrategias tempranas de prevención y control. De hecho, para el departamento del Atlántico se mantiene un estricto esquema para la ampliación de las coberturas de vacunación antiamarílica, con base en el hallazgo de $H$. equinus en áreas periurbanas de Soledad y Malambo, y ante los altos índices de infestación por $A$. aegypti, especie que ha sido incriminada como vector urbano de fiebre amarilla en Colombia (33).

También se han definido nuevos sitios de vigilancia entomológica mediante larvitrampas (centro y sur del departamento), se han gestado estrategias coordinadas con el departamento del Magdalena (endémico para fiebre amarilla de ciclo selvático) para la vigilancia activa en municipios limítrofes entre los dos departamentos y la búsqueda activa de $H$. equinus en el intradomicilio de los barrios cercanos al hallazgo de esta especie en Soledad y Malambo.

En la costa atlántica colombiana se tiene registro de $H$. equinus en el departamento de La Guajira, donde se encontró adaptada a la vegetación cactácea (16). Con los resultados de este trabajo se contribuye a la ampliación de su distribución y bionomía.

\section{Agradecimientos}

Expresamos agradecimientos a Mariela Vergara $y$ todos los funcionarios de aeropuertos del Caribe S.A. (aeropuerto Ernesto Cortissoz); así como a las autoridades y comunidad del Batallón Vergara y Velasco por la colaboración brindada en la realización de la vigilancia entomológica. Agradecemos a Luis Carlos Guzmán, técnico del Programa de Enfermedades Transmitidas por Vectores, por su colaboración en la recolección del material entomológico.

A Víctor Olano, Investigador Emérito del Laboratorio de Entomología del Instituto Nacional de Salud por las confirmaciones taxonómicas y controles de calidad del material entomológico remitido. Al biólogo Jorge de las Salas Alí por la asesoría en el manejo del programa Arcview, para 
la realización de los mapas del departamento del Atlántico y sus aportes académicos para la realización de este trabajo.

A Alberto Morales Alarcón MSc, Investigador Emérito del Instituto Nacional de Salud por los aportes académicos acerca de la actualización de especies del genero Haemagogus registradas para Colombia.

\section{Conflicto de intereses}

Los autores declaramos que no existe conflicto de intereses que puedan afectar los resultados de esta investigación.

\section{Financiación}

El presente trabajo fue financiado por la Gobernación del Atlántico-Secretaría de Salud (Subsecretaría de Salud Pública).

\section{Referencias}

1. Vasconcelos da Costa PF. Febre Amarela. Rev Soc Bras Med Trop. 2003;36:275-93.

2. Tesh RB. Viral hemorrhagic fevers of South America. Biomédica. 2002;22:287-95.

3. Rodríguez A, Rodríguez R, Granados R, Villareal L, Pérez L, Olano V, et al. Fiebre amarilla en Colombia. Inf Quinc Epidemiol Nac. 1996;1:1-7.

4. Rodríguez G, Velandia M, Boshell J. Fiebre amarilla: la enfermedad y su control. Bogotá D.C.: Instituto Nacional de Salud; 2003. p.40.

5. Méndez JA, Rodríguez G, Bernal MP, Calvache D, Boshell J. Detección molecular del virus de la fiebre amarilla en muestras de suero de casos fatales humanos y en cerebro de ratón. Biomédica. 2003;23:232-8.

6. Oyewale T. Fiebre amarilla en África: su impacto en salud pública y perspectivas para su control en el siglo XXI. Biomédica. 2002;22:194-210.

7. Dunster L, Sanders EJ, Borus P. Tukei PM. Yellow fever in Kenya: the need for a country-wide surveillance program. World Health Stat Q. 1997;50:178-84.

8. PAHO. Present status of yellow fever. Memorandum from a PAHO meeting. Bull World Health Organ. 1986;64:511-24

9. Aitken TH, Tesh RB, Beaty BJ, Rosen L. Transovarial transmission of yellow fever virus by mosquitoes (Aedes aegypti). Am J Trop Med Hyg. 1979;28:119-21.

10. Kumm H, Osorno E, Boshell J. Studies on mosquitoes of the genus Haemagogus in Colombia (Diptera: Culicidae). Biomédica. 1996;16:269-92.
11. Morales A. Distribución geográfica, horizontal y vertical de Haemagogus (Diptera: Culicidae) de Colombia. Rev Acd Col Ciencias Exactas Fis Nat. 1968;13:267-72.

12. Knight K, Stone A. A catalog of the mosquito of the world (Diptera: Culicidae). Second Edition. USA: The Thomas Say Foundation; 1977. p.179-81.

13. Arnell JH. Mosquito Studies (Diptera, Culicidae). XXXII. A revision of the genus Haemagogus . Contr Am Ent Inst.1973;10:1-174.

14. Zavortink TJ. Mosquito studies (Diptera, Culicidae). XXVII.The New World species formerly placed in Aedes (Finlaya). Contr Am Ent Inst.1972;8:206.

15. Forattini OP. Entomología Médica. Volumen II. Sao Paulo: Editora da Universidade;1965.p.378-82.

16. Morales A, Ferro de Carrasquilla C, Isaza de Rodríguez C, Cura E. Búsqueda de mosquitos del género Haemagogus en el departamento de la GuajiraColombia, Sur América (Diptera: Culicidae). Biomédica. 1984;4:25-33.

17. Olano V, Bueno A, González C, Correa M. Hallazgo del mosquito Haemagogus equinus (Theobald, 1903) en el área metropolitana de Bucaramanga, Santander. Biomédica. 1997;17:128-31.

18. Escobar J, López Y, Osorio L, González M, Wolff M. Manual para la vigilancia y control de vectores de malaria, dengue, fiebre amarilla, leishmaniasis, enfermedad de Chagas y encefalitis equina venezolana desde el nivel municipal. Medellín: Dirección Seccional de Antioquia; 1999. p.93-7.

19. Organización Mundial de la Salud. Fiebres hemorrágicas víricas. Ginebra: WHO; 1985. p.45.

20. Bres P. Century of progress in combating yellow fever. Bull WHO. 1986;54:775-861.

21. Secretaría de Salud Municipal de Soledad. Plan de Ordenamiento Territorial (POT). Soledad: Secretaría de Salud Municipal; 2001. p.15-20.

22. Corporación Autónoma Regional del Atlántico. Evaluación del estado actual de la flora y fauna en los municipios de Soledad y Malambo. Barranquilla: Corporación Autónoma Regional del Atlántico; 2002. p.87-125.

23. Secretaría de Salud Municipal de Malambo. Plan de Ordenamiento Territorial de Malambo (POT). Tomo IV. Documento Técnico Parte A. Componente General Urbano y Rural. Malambo: Secretaria de Salud Municipal; 2001. p.48-60.

24. Secretaría de Salud Municipal de Malambo. Plan de Ordenamiento Territorial de Malambo (POT). Tomo II: Documento Técnico Parte A. Diagnóstico: subsistema biofísico y funcionamiento espacial. Malambo: Secretaría de Salud Municipal; 2001. p.85-92.

25. Cova García P, Sutil E, Rausseo JA. Mosquitos de Venezuela. Tomo I. Caracas: Ministerio de Sanidad y Asistencia Social; 1966. p.410. 
26. Tinker M. Clave práctica para las larvas de mosquitos neotropicales en recipiente. Bogotá, D.C: Instituto Nacional de Salud; 1982. p.1-15.

27. Rueda LM. Pictorial keys for the identification of mosquitoes (Diptera: Culicidae) associated with dengue virus transmisión. Zootaxa. 2004;589:1-60.

28. Forattini O, Kakitani I, Mureb Sallum M. Encontro de criadouros de Aedes scapularis (Diptera: Culicidae) em recipientes artificiales. Rev Saude Publica. 1997;31:519-22.

29. Velandia M, Vera M, García I, Bernal M, Méndez J, Olano V, et al. Vigilancia epidemiológica de los eventos prevenidos por el Programa Ampliado de Inmunización,
Colombia, 2003: fiebre amarilla. Inf Quinc Epidemiol Nac. 2004;9:145-60.

30. Instituto Nacional de Salud. Sistema Nacional de Vigilancia en Salud Pública, SIVIGILA 1997-2005. Bogotá, D.C.: Instituto Nacional de Salud; 2005.

31. Vera N. Situación de la fiebre amarilla en Colombia, semanas 1 a 35 de 2005. Inf Quinc Epidemiol Nac. 2005;10:241-6.

32. Olano V, González C. Vigilancia entomológica para Aedes albopictus. Inf Quinc Epidemiol Nac. 1997;2:93-4.

33. Gast G. Historia de la fiebre amarilla en Colombia. Bogotá: Instituto Nacional de Salud; 1982. 